\title{
Relating optical and microwave grain metrics of snow: the relevance of grain shape
}

\author{
Quirine Krol and Henning Löwe \\ WSL Institute for Snow and Avalanche Research SLF, Flüelastrasse 11, 7260 Davos Dorf, Switzerland \\ Correspondence to: Henning Löwe (loewe@slf.ch)
}

Received: 13 May 2016 - Published in The Cryosphere Discuss.: 10 June 2016

Revised: 31 October 2016 - Accepted: 2 November 2016 - Published: 21 November 2016

\begin{abstract}
Grain shape is commonly understood as a morphological characteristic of snow that is independent of the optical diameter (or specific surface area) influencing its physical properties. In this study we use tomography images to investigate two objectively defined metrics of grain shape that naturally extend the characterization of snow in terms of the optical diameter. One is the curvature length $\lambda_{2}$, related to the third-order term in the expansion of the two-point correlation function, and the other is the second moment $\mu_{2}$ of the chord length distributions. We show that the exponential correlation length, widely used for microwave modeling, can be related to the optical diameter and $\lambda_{2}$. Likewise, we show that the absorption enhancement parameter $B$ and the asymmetry factor $g^{\mathrm{G}}$, required for optical modeling, can be related to the optical diameter and $\mu_{2}$. We establish various statistical relations between all size metrics obtained from the two-point correlation function and the chord length distribution. Overall our results suggest that the characterization of grain shape via $\lambda_{2}$ or $\mu_{2}$ is virtually equivalent since both capture similar aspects of size dispersity. Our results provide a common ground for the different grain metrics required for optical and microwave modeling of snow.
\end{abstract}

\section{Introduction}

Linking physical properties of snow to the microstructure always requires the identification of appropriate metrics of grain size. In this regard the two-point correlation function has become a key quantity for the prediction of various properties such as thermal conductivity, permeability and electromagnetic properties of snow (Wiesmann and Mätzler, 1999; Löwe et al., 2013; Calonne et al., 2014b; Löwe and Pi- card, 2015). The two-point correlation function carries, in essence, information about a distribution of relevant sizes in the microstructure. For microwave applications, the analysis of two-point correlation functions was already used in the era before micro-computed tomography $(\mu \mathrm{CT})$, where thin section data and stereology were employed to obtain the required information (Vallese and Kong, 1981; Zurk et al., 1997; Mätzler and Wiesmann, 1999). The recently gained interest in two-point correlation functions is mainly driven by available data from $\mu \mathrm{CT}$, from which the two-point correlation function can be conveniently estimated. The relevance of the two-point correlation function for microwave modeling originates from the connection between its Fourier transform and the scattering phase function in the Born approximation for small scatterers (Mätzler, 1998; Ding et al., 2010; Löwe and Picard, 2015) or the connection to the effective dielectric tensor via depolarization factors (Leinss et al., 2016).

A common practical way to characterize the two-point correlation function is a fit to an exponential, such that the fit parameter, the so-called exponential correlation length $\xi$, can be used to model the decay of microstructural correlations in snow by a single size parameter. This approach dates back to Debye et al. (1957) in the context of small angle scattering of heterogeneous materials. However, the characterization of snow by a single length $\xi$ is only an approximation since the occurrence of multiple length scales (Löwe et al., 2011) is known to play a role, in particular to characterize anisotropy (Löwe et al., 2013; Calonne et al., 2014b). Despite this caveat, $\xi$ still constitutes the main microstructural parameter for microwave modeling of snow (Proksch et al., 2015a; Pan et al., 2016) when the Microwave Emission Model of Layered Snowpacks (MEMLS) (Wiesmann et al., 1998 ) is used. 
The exponential correlation length is often inferred from measurements of the optical equivalent diameter $d_{\mathrm{opt}}$ or, equivalently, from the specific surface area (SSA). This link was established statistically (Mätzler, 2002), leading to the empirical relation

$\xi \approx 0.5 d_{\text {opt }}(1-\phi)$,

where $\phi$ is the ice volume fraction. This relation facilitates using the measured optical diameter as the primary input for microwave modeling (Durand et al., 2008; Proksch et al., 2015b; Tan et al., 2015). However, this link between $\xi$ and $d_{\text {opt }}$ can only serve as a first approximation. The numerical prefactor in Eq. (1) seems to depend on snow type (Mätzler, 2002), which causes a significant scatter in estimating the exponential correlation length from optical diameter. This poses the question of which additional size metric captures variations in grain shape and explains the scatter.

A similar issue of grain shape emerges in the context of optical measurements. Optical properties (e.g., reflectance) can be largely predicted from the optical diameter or SSA (Kokhanovsky and Zege, 2004). The remaining scatter is commonly attributed to shape (Picard et al., 2009), which influences the absorption enhancement parameter $B$ and the asymmetry factor $g^{\mathrm{G}}$ (Kokhanovsky and Zege, 2004). The influence of grain shape on $B$ for light penetration was recently addressed and measured by Libois et al. $(2013,2014)$. The question remains of which additional size metric of the microstructure can be used to capture variations in grain shape and measured scatter in $B$.

The two examples from microwave or optical modeling above reflect the known fact that the optical diameter as a single metric of grain size is not sufficient to characterize the microstructure for many physical properties. It is thus necessary to account for additional grain size metrics which implement the idea of grain shape. A key requirement for potential new shape metrics is a well-defined geometrical meaning. Present snowpack models (Vionnet et al., 2012; Lehning et al., 2002) contain empirical shape descriptors such as sphericity (Brun et al., 1992). An objective definition of these quantities for arbitrary two-phase materials is, however, not possible. New shape metrics should thus ideally seek to replace empirical parameters by an objective, measurable and geometrically comprehensible metric.

One appealing route to define shape is via curvatures of the ice-air interface because curvatures (i) have already been used to comprehend snow metamorphism via mean and Gaussian curvatures (Brzoska et al., 2008; Schleef et al., 2014; Calonne et al., 2014a), (ii) are natural quantities to assess shape via deviations from a sphere, very close to the definition of sphericity in Lesaffre et al. (1998), and (iii) naturally emerge as higher-order terms in the expansion of the two-point correlation function (Torquato, 2002). The latter fact can be used in turn to assess variations of the microwave parameter $(\xi)$ from $\mu \mathrm{CT}$ images, which links back to the aforementioned microwave modeling problem.
Another appealing route to define shape is via chord length distributions because they (i) naturally implement the idea of size dispersity and (ii) have recently been put forward by Malinka (2014) to derive closed-form expressions for the averaged optical properties of a porous medium. Again, the latter fact can in turn be used to assess variations in the optical parameters $\left(g^{\mathrm{G}}, B\right)$ from $\mu \mathrm{CT}$ images, which links back to the aforementioned optical modeling problem.

The motivation of the present paper is to investigate and interconnect these two routes of (objectively) defining grain shape. First, we will assess the curvature length in the expansion of the two-point correlation function. We will be guided by the questions of whether, and how, the well-known statistical relation Eq. (1) between the exponential correlation length and the optical diameter can be improved by incorporating curvatures. Second, we will characterize the microstructure in terms of chord length distributions in order to make contact to aspects of shape in snow optics. An interconnection between the two routes can be established by an approximate relation between the two-point correlation function and the chord length distribution that was originally suggested in the context of small angle scattering (Méring and Tchoubar, 1968). By means of this approximate relation we establish various statistical links between all involved size metrics, the moments of the chord length distributions, optical diameter, surface areas, curvatures and the exponential correlation length. The established links imply a microstructural connection between geometrical optics and microwave scattering via size dispersity, which constitutes one aspect of grain shape.

The paper is organized as follows. In Sect. 2 we present the theoretical background for the two-point correlation function, the chord length distribution, the connection between both quantities and the governing length scales. In Sect. 3 we provide a summary of the $\mu \mathrm{CT}$ image analysis methods. To provide confidence of the interpretation of the curvature metrics derived from the two-point correlation function, we present an independent validation of these quantities via the triangulation of the ice-air interface. The results of the statistical models are presented in Sect. 4 and discussed in Sect. 5.

\section{Theoretical background}

\subsection{Two-point correlation function and microwave metrics}

The interaction of microwaves with snow is commonly interpreted as scattering at permittivity fluctuations in the microstructure, which can be described by the two-point correlation function (Vallese and Kong, 1981; Mätzler, 1998; Ding et al., 2010; Löwe and Picard, 2015). The two-point correlation function can be derived from the spatial distribution of ice and air that is characterized by the ice-phase indicator function $\mathcal{I}(\boldsymbol{x})$, which is equal to 1 for a point $\boldsymbol{x}$ in ice and 
0 for $\boldsymbol{x}$ in air. From that, a covariance function can be defined, which is often referred to as the two-point correlation function:

$C(\boldsymbol{r})=\overline{\mathcal{I}(\boldsymbol{x}+\boldsymbol{r}) \mathcal{I}(\boldsymbol{x})}-\phi^{2}$.

In the following we disregard anisotropy by stating that $C(r)$ only depends on the magnitude of $r=|\boldsymbol{r}|$. To interpret snow with this approach, an average over different coordinate directions must be carried out.

The value of the two-point correlation function $C(0)=$ $\phi(1-\phi)$ is simply related to the volume fractions of ice and air. Therefore, often only the normalized two-point correlation function is used (see Fig. 1b):

$A(r)=C(r) / C(0)$.

Since $A(r)$ must decay from $A(0)=1$ to 0 for $r \rightarrow \infty$, the two-point correlation function is often described by an exponential form

$A(r)=\exp (-r / \xi)$

in terms of the exponential correlation length $\xi$. This single length scale empirically characterizes the decay of $A(r)$.

For small arguments $r$, rigorous results for the decay of the correlation can also be inferred since the expansion of $A(r)$ can be interpreted in terms of geometrical properties of the interface. According to Torquato (2002), the expansion for an isotropic medium reads

$A(r)=1-\frac{r}{\lambda_{1}}\left[1-\frac{r^{2}}{\lambda_{2}^{2}}+\mathcal{O}\left(r^{3}\right)\right]$

in terms of the length scales $\lambda_{1}$ and $\lambda_{2}$. The first-order term

$\frac{1}{\lambda_{1}}=-\left.\frac{\mathrm{d}}{\mathrm{d} r} A(r)\right|_{r=0}=\frac{s}{4 \phi(1-\phi)}$

is the slope of the two-point correlation function at the origin and can be expressed in terms of the interfacial area per unit volume $s$ (Debye et al., 1957). The size metric $\lambda_{1}$ is one of the most fundamental length scales for a two-phase medium and referred to as the "Porod length" in small angle scattering, or "correlation length" in Mätzler (2002). We will adhere to Porod length here to clearly distinguish $\lambda_{1}$ from the exponential correlation length $\xi$. The metric $\lambda_{1}$ can be also related to the SSA, defined as the surface area per ice mass $\left(\mathrm{m}^{2} \mathrm{~kg}^{-1}\right)$, or in turn to the equivalent optical diameter $d_{\mathrm{opt}}$ of snow via

$\lambda_{1}=\frac{4 \phi(1-\phi)}{s}=\frac{4(1-\phi)}{\rho_{\mathrm{i}} \mathrm{SSA}}=\frac{2(1-\phi)}{3} d_{\mathrm{opt}}$,

with $\rho_{\mathrm{i}}$ representing the density of ice. The last equality is obtained when the definition of $d_{\mathrm{opt}}=6 / \rho_{\mathrm{i}} \mathrm{SSA}$ is inserted (see Mätzler, 2002).
For a two-phase material with a smooth interface, the second-order term $\sim r^{2}$ is missing in the expansion Eq. (5) and the next non-zero term is the cubic one with a prefactor $1 / \lambda_{1} \lambda_{2}^{2}$. Here the length scale $\lambda_{2}$ has a geometric interpretation in terms of interfacial curvatures and is therefore referred to as the curvature length hereafter. As originally shown by Frisch and Stillinger (1963), the following identity holds

$\frac{1}{\lambda_{2}^{2}}=\left.\lambda_{1} \frac{\mathrm{d}^{3}}{\mathrm{~d} r^{3}} A(r)\right|_{r=0}=\frac{1}{8}\left(\overline{H^{2}}-\frac{\bar{K}}{3}\right)$

in terms of the average squared-mean curvature $\overline{H^{2}}$ and the averaged Gaussian curvature $\bar{K}$. The quantity $\lambda_{2}^{-2}$ is proportional to the orientationally averaged normal curvature of an interface (Tomita, 1986).

\subsection{Chord length distributions and optical metrics}

In snow optics the microstructural characterization within radiative transfer theory (Kokhanovsky and Zege, 2004) commonly involves a single metric, the optical diameter. An interesting approach for geometrical optics in arbitrary twophase media was recently put forward by Malinka (2014). Thereby, the microstructure is taken into account by the chord length distribution of a medium which can be unambiguously defined for arbitrary two-phase random media (Torquato, 2002). Chord lengths in an isotropic medium are defined as the lengths of the intersections of random rays through the sample with the ice phase, as illustrated in the schematic in Fig. 1a. The chord length distribution $p(\ell)$ of the ice phase denotes the probability (density) for finding a chord of length $\ell$.

In contrast to the Born approximation for microwaves, where the microstructure enters as the Fourier transform of the two-point correlation function, the theoretical approach (Malinka, 2014) relates the key optical quantities (absorption, phase function, asymmetry factor) to the Laplace transform of the chord length distribution $p(\ell)$ which is denoted by

$\widehat{p}(z)=\int_{0}^{\infty} \mathrm{d} \ell p(\ell) \mathrm{e}^{-z \ell}$

with Laplace variable $z$. For small $z$, the Laplace transform can be approximated by the expansion

$\widehat{p}(z)=1-\mu_{1} z+\frac{\mu_{2}}{2} z^{2}+\mathcal{O}\left(z^{3}\right)$,

where $\mu_{i}$ denotes the $i$ th moment of the chord length distribution, viz.

$\mu_{i}=\int_{0}^{\infty} \mathrm{d} \ell \ell^{i} p(\ell)$. 
(a)

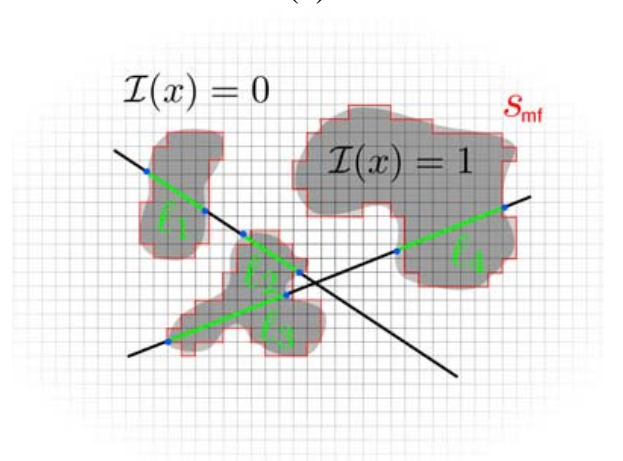

(b)

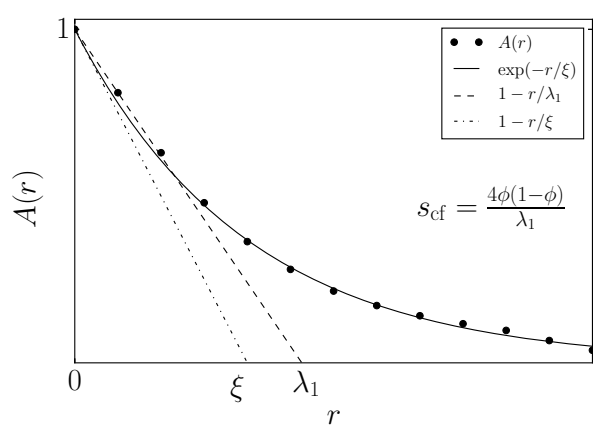

Figure 1. (a) Illustration of the chord lengths obtained from an ice sample. The mean chord length is defined as the average length of the green line lengths. A stereological approach (Underwood, 1969) to calculate $s$ is to count the number of blue dots per unit length. The estimation for $s_{\mathrm{mf}}$ is given by the red contour. (b) Illustration of the two-point correlation function $A(r)$ and the method obtaining an estimate for the Porod length $\lambda_{1}$ to get $s_{\mathrm{cf}}$ by fitting the slope at the origin, and the exponential correlation length $\xi$ by fitting $A(r)$ to $\exp (-r / \xi)$ over a larger span.

Hence, within the approach from Malinka (2014), the optical response of snow can be systematically improved by successively including higher moments of the chord length distribution. According to Malinka (2014), the Laplace transform has to be evaluated at $z=\alpha$, with the absorption coefficient $\alpha=4 \pi \kappa / \lambda$. Here $\lambda$ is the wavelength and $\kappa$ the imaginary part of the refractive index of ice. It is generally sufficient (Malinka, 2014) to retain only a few terms in Eq. (10). It is straightforward to show (Underwood, 1969) that the first moment, i.e, the mean chord length $\mu_{1}$, is given by

$\mu_{1}=\frac{4 \phi}{s}=\frac{\lambda_{1}}{1-\phi}=\frac{2}{3} d_{\mathrm{opt}}$

and thus related to the surface area per unit volume $s$ from Eq. (6), or the optical diameter $d_{\text {opt }}$ via Eq. (7). Therefore, in lowest order, the Laplace transform Eq. (9) only contains the Porod length or specific surface area of snow. The next order correction involves the second moment $\mu_{2}$ for which no geometric interpretation has been hitherto given for arbitrary two-phase random media.
For known chord length distribution, all optical quantities (phase function, single scattering albedo, etc.) can be directly computed from Malinka (2014). To make contact to other approaches, for example Libois et al. (2013), and discuss our results for the chord lengths in light of shape, an expression of the absorption enhancement parameter $B$ is required within the framework of Malinka (2014), which is derived in Appendix A. From these expressions we can asses the relative importance of the $\mu_{2}$ correction to the optical diameter $\mu_{1}$.

\subsection{Connection between chord lengths and the Porod length and the curvature length}

Following the previous two sections, a link between optical and microwave metrics of snow thus requires to establish a link between two-point correlation functions and chord length distributions. To this end we employ a relation between the two-point correlation function and chord length distribution that was put forward in the early stages of small angle scattering (Méring and Tchoubar, 1968) to interpret the scattering curve in terms of particle properties. In the present notation the relation can be written as

$p(\ell)=\mu_{1} \frac{\mathrm{d}^{2}}{\mathrm{~d} \ell^{2}} A(\ell)$,

which was also used by Gille (2000).

Although Eq. (13) is only valid under certain assumptions which will be discussed in Sect. 5, it has already some non-trivial implications that can be exploited for the subsequent analysis. As a first consistency check of the approximation Eq. (13), we can compute the first moment of the chord length distribution from Eq. (11) for $n=1$ by inserting Eq. (13) and integrating by parts. This yields $\mu_{1}=\mu_{1} A(0)$, which is correct by virtue of Eq. (3). As a next step, we aim at an expression for the second moment of the chord length distribution in terms of interfacial curvatures by using Eq. (11) for $n=2$. Again, inserting Eq. (13) and integrating by parts yields

$\mu_{2}=2 \mu_{1} \int_{0}^{\infty} A(r) \mathrm{d} r=2 \mu_{1} f\left(\phi, \lambda_{1}, \lambda_{2}, \ldots\right)$.

Though $f$ is an unknown function here, this link shows that the chord length metric $\mu_{2}$ must be somehow related to the two-point correlation function metrics $\lambda_{1}$ and $\lambda_{2}$. In Sect. 4 we will statistically investigate the dependence of $f$ on its arguments.

\section{Methods}

\subsection{Data}

For the following analysis we used an existing $\mu \mathrm{CT}$ data set of 3-D microstructure images described and used in Löwe 
et al. (2013) for a thermal conductivity analysis and Löwe and Picard (2015) for a comparison of microwave scattering coefficients. All samples were classified according to Fierz et al. (2009) as described in the supplement of Löwe et al. (2013). The data set comprises 167 different samples including two time series of isothermal experiments, four time series of temperature gradient metamorphism experiments and a set of 37 individual samples. In total, the set includes 62 samples of depth hoar (DH), 54 of rounded grains (RG), 33 of faceted crystals (FC) 10 of decomposing and fragmented precipitation particles (DF), 5 of melt forms (MF) and 3 of precipitation particles (PP).

\subsection{Geometry from two-point correlation functions}

Obtaining the normalized two-point correlation function $A(r)$ from a $\mu \mathrm{CT}$ image can be conveniently done by using the fast Fourier transform (FFT) as, for example, described in Newman and Barkema (1999). The FFT is typically used for performance issues to evaluate the convolution integral Eq. (2) since direct methods can be very slow. The spatial resolution of the two-point correlation function depends on the voxel size $\Delta$ of the $\mu \mathrm{CT}$ image which ranges from 10 to $50 \mu \mathrm{m}$.

Since the snow samples in the data set are anisotropic (Löwe et al., 2013), the normalized two-point correlation function is first obtained in the $x, y$ and $z$ direction and then averaged arithmetically over the three directions, i.e., $A(r)=$ $\left(A_{x}(r)+A_{y}(r)+A_{z}(r)\right) / 3$.

From the normalized two-point correlation function two types of parameter fittings are performed. First, the exponential correlation length $\xi$ is obtained by fitting the $\mu \mathrm{CT}$ data to the exponential form Eq. (4). Technically, we estimated the inverse parameter $k$ by least-squares optimization of the model $A(r)=\exp (-k r)$ to the data in a fixed range of $0<$ $r<50 \Delta$. An illustration of this method is shown in Fig. $1 \mathrm{~b}$. In the following we denote by $\xi$ the inverse of the optimal fit parameter $\xi:=1 / k$. Secondly, we estimated the expansion parameters $\lambda_{1}$ and $\lambda_{2}$ of the two-point correlation function by a least-squares regression to the expansion Eq. (5). Technically, we fitted $A(r)=1-k_{1} r\left(1-k_{2} r^{2}\right)$ in the fixed range of $0<r<3 \Delta$, which determines the derivatives at the origin. We denote by $\lambda_{1}^{\mathrm{cf}}$ and $\lambda_{2}^{\mathrm{cf}}$ the inverse of the optimal fit parameters $\lambda_{1}^{\mathrm{cf}}:=1 / k_{1}$ and $\lambda_{2}^{\mathrm{cf}}:=1 / k_{2}$. The superscript is added to discern these two-point correlation-function-based estimates from those presented in the next section for a validation. The influence of resolution and anisotropy to the estimates of $\lambda_{1}$ and $\lambda_{2}$ is discussed in Sect. 5 .

\subsection{Geometry from triangulations}

To confirm the geometrical interpretation of $\lambda_{1}^{\mathrm{cf}}$ and $\lambda_{2}^{\mathrm{cf}}$ we use an alternative and independent method to estimate these parameters by measuring the surface area and the local curvatures with a VTK-based image analysis as described in Krol and Löwe (2016). In short, a triangulated ice-air interface is obtained by applying the VTKContour filter. After this step, the interface still resembles the underlying voxel structure. Therefore, in a second step the triangulated interface is smoothed by applying the VTKSmoothing filter, which involves a smoothing parameter $S$ that is the number of iterations a Laplacian smoothing on a mesh is repeated. For further details we refer to Krol and Löwe (2016).

\subsection{Accuracy of surface area and curvatures estimates}

The measured total surface area is obtained by integrating (summing) the surface area of the triangles over the surface and the estimate $\lambda_{1}^{\text {vtk }}$, which naturally depends on the smoothing parameter. A comparison of the triangulation and the two-point correlation-function-based length scale is shown in Fig. 2 (middle row). A higher value of the smoothing parameter implies a lower surface area $s$ (caused by shrinking of the enclosed volume upon smoothing) and in

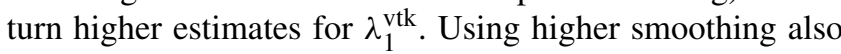
results in a higher variance in the data. This is likely due to filtering of small perturbations in the surface causing the individual samples to react differently.

It is illustrative to note that even without smoothing for $S=0$ the obtained triangulated surface is still different from the voxel surface $s_{\mathrm{mf}}$, which is obtained by the union of ice-air transition faces in the voxel-based image (as illustrated by the red contour in Fig. 1a). The quantity $s_{\mathrm{mf}}$ is one of the four Minkowski functionals and can be computed by standard counting algorithms (Michielsen and Raedt, 2001). For isotropic systems, as well as statistically representative samples, the relation between the surface obtained from the two-point correlation function $s_{\mathrm{cf}}=4 \phi(1-\phi) / \lambda_{1}^{\mathrm{cf}}$ and the Minkowski functionals is known to be $s_{\mathrm{cf}}=2 s_{\mathrm{mf}} / 3$ as discussed in Torquato (2002, p. 290).

An estimate for the curvature-length $\lambda_{2}^{\mathrm{vtk}}$ is obtained from the VTKCurvature filter on the triangulated ice-air interface yielding local values for mean and Gaussian curvature which can be integrated to compute $\lambda_{2}^{\text {vtk }}$ via Eq. (8). The comparison of the triangulation-based curvature length and the twopoint correlation-function-based curvature length is shown in Fig. 2 (bottom row). Again, $\lambda_{2}^{\text {vtk }}$ depends strongly on the smoothing parameter $S$. The value $S=200$ performed best

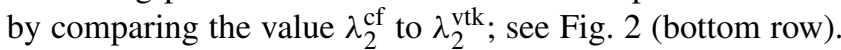
The deviations from the $1: 1$ line are caused by the overestimation of the curvatures by the remaining steps in the triangulation from the underlying voxel-based data and is thus negatively correlated with the size of the structures and the resolution. In the end, we chose a smoothing parameter $S=200$ that is, on average, acceptable for all involved samples.

Overall, the comparison provides reasonable confidence that the geometrical interpretation of the two-point correlation function parameters is correct, though uncertainties inherent to the smoothing operations must be acknowledged. In 

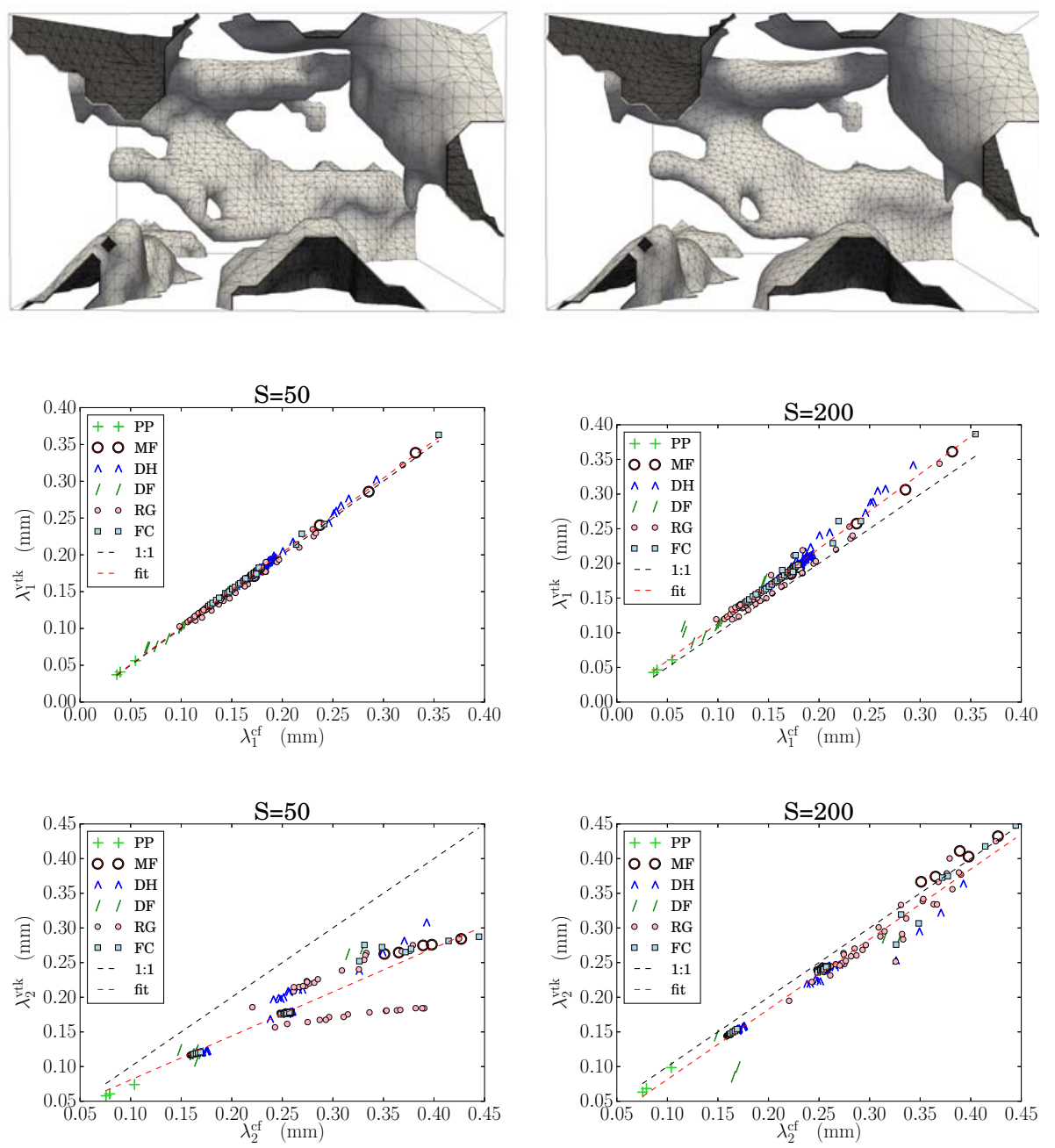

Figure 2. Comparison between smoothing parameter $S=50$ (left) and $S=200$ (right). Top: representation of the triangulated surface of a subsection of a snow sample. Middle: scatterplots of the Porod length $\lambda_{1}^{\text {cf }}$ versus $\lambda_{1}^{\text {vtk}}$, including a fit (red dotted line). Bottom: scatterplots of the curvature-length $\lambda_{2}^{\mathrm{cf}}$ versus $\lambda_{2}^{\mathrm{vtk}}$, including a fit (red dotted line).

the following we solely use the quantities derived from the two-point correlation function, viz. $\lambda_{1}=\lambda_{1}^{\mathrm{cf}}$ and $\lambda_{2}=\lambda_{2}^{\mathrm{cf}}$, where the superscripts are omitted for brevity.

\subsection{Chord length distribution}

To compute the ice chord length distribution from the binary images, all linear lines through the sample in all three Cartesian directions $\beta=x, y, z$ are considered and all ice chords were measured and binned to obtain direction dependent counting densities $n^{\beta}(\ell)$. Here $n^{x}(\ell)$ denotes the total number of chords in $x$ direction, which have length $\ell$. For a binary CT image, $\ell$ can take integer values $0<\ell<L_{x}$ which are restricted by the sample size $L_{x}=N_{x} \Delta$ and the voxel size $\Delta$ of the image. The mean chord length and other moments $\mu_{i}$ are then computed from $\mu_{i}=\frac{1}{\sum_{\ell, \beta} n^{\beta}(\ell)} \sum_{\ell, \beta} \ell^{i} n^{\beta}(\ell)$.

\subsection{Statistical models}

The main part of the following analysis comprises statistical relations between the length scales derived from the chord length distribution and the two-point correlation function in Sect. 2. In total, we will consider a few statistical models that first relate the exponential correlation length $\xi$ and $\mu_{2}$ to the geometrical length scales $\lambda_{1}$ and $\lambda_{2}$ and second, relate $\xi$ to $\mu_{1}$ and $\mu_{2}$. We will start with a one-parameter statistical model and compare the results to the two-parameter models. We will assess and compare the quality of the fits with the adjusted correlation coefficient $R^{2}$. 


\section{Results}

\subsection{Relating exponential correlation length to the Porod length and curvature length}

As a starting point for the statistical analysis we revisit the empirical relation

$\xi=0.75 \lambda_{1}$,

which is equivalent to Eq. (1) by virtue of Eq. (7), as suggested by Mätzler (2002). To this end we fitted $\xi$ and $\lambda_{1}$ and obtained an average slope of 0.79 with a correlation coefficient of $R^{2}=0.733$, shown by the green dashed line in Fig. 3a. In the next step we fitted the same data to include an intercept parameter

$\xi=a_{0}+a_{1} \lambda_{1}$.

Here the adjusted correlation coefficient, accounting for the inclusion of extra parameters, is $R^{2}=0.731$ and the parameters are given by $a_{0}=5.93 \times 10^{-2} \mathrm{~mm}$ and $a_{1}=0.794$, with very low $p$ values $\left(p<5 \times 10^{-4}\right)$ for the intercept and the slope ensuring the significance of the parameters of the fit. The order of magnitude of the intercept $a_{0}$ is negligible. To understand the remaining scatter we have plotted the residuals $\xi-\left(a_{0}+a_{1} \lambda_{1}\right)$ versus the curvature-length $\lambda_{2}$ as shown in Fig. 3b. The correlation coefficient is given by $R^{2}=0.644$ and suggests that including the curvature lengths can improve Eq. (17). For an overview, this and all other statistical models are listed in Table 1.

In the next step we include the curvature-length $\lambda_{2}$ where we fitted the exponential correlation length $\xi$ to the model

$\xi=b_{0}+b_{1} \lambda_{1}+b_{2} \lambda_{2}$.

The results are shown in Fig. 3c. Here we find an improvement compared to Eq. (17). The correlation coefficient is $R^{2}=0.922$ and the fit parameters are given by $b_{0}=1.23 \times$ $10^{-2} \mathrm{~mm}, b_{1}=1.32$ and $b_{2}=-3.85 \times 10^{-1}$. The $p$ values are very small for all coefficients $b_{i}$. The order of magnitude of the improvement can already be roughly estimated from the ratio of the prefactors $b_{1}$ and $b_{2}$.

\subsection{Connection between chord length distributions and two-point correlation functions}

To relate the chord length metrics to the Porod length and the curvature length, we first assessed the relation between the chord length distribution $p(\ell)$ and the two-point correlation function $A(\ell)$ as suggested by Eq. (13). To this end we compared the chord length distribution obtained directly from the $\mu \mathrm{CT}$ image (cf. Sect. 3.5) with the prediction of Eq. (13) via the two-point correlation function for a few examples of different snow types. The results are shown in Fig. 4. The selected snow samples are the same as those used in Löwe and Picard (2015, Figs. 8 and 9). Qualitatively, the characteristic (a)

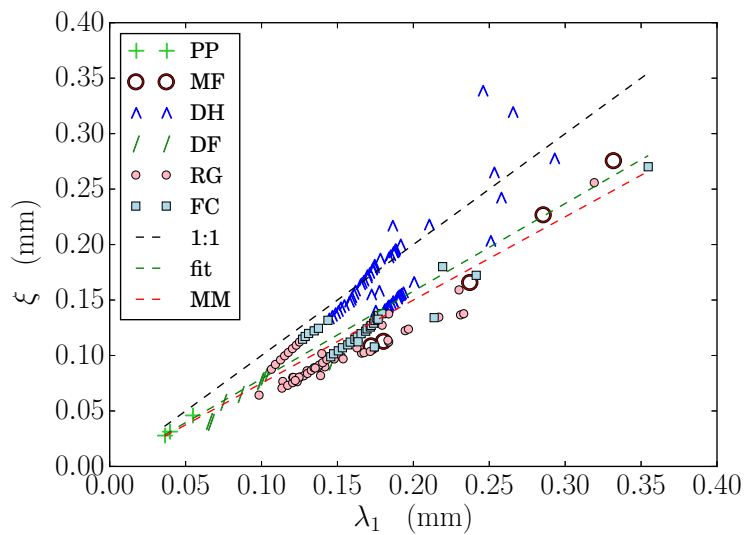

(b)

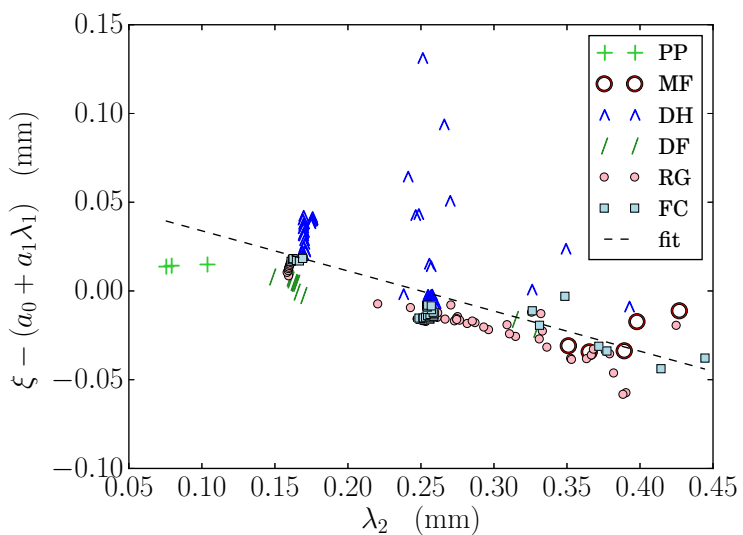

(c)

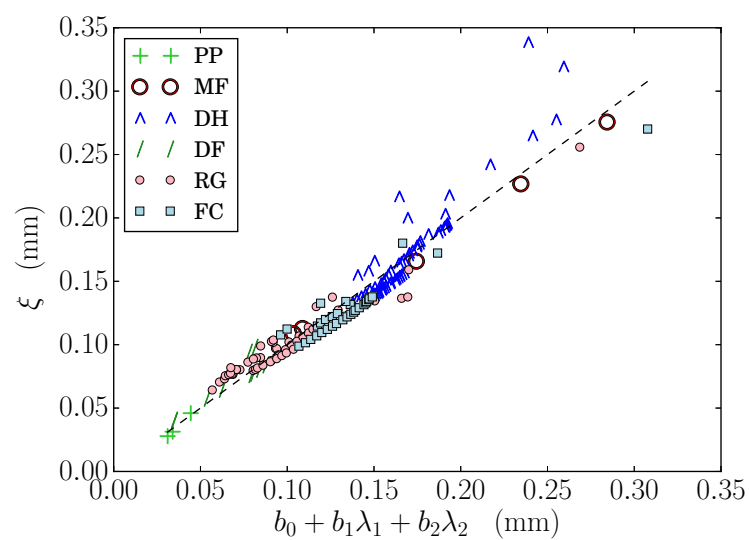

Figure 3. Scatterplots of (a) the exponential correlation length $\xi$ versus the Porod length $\lambda_{1}$. A linear fit is plotted in green. Additionally, the prediction of Eq. (16) (MM) is plotted in red. (b) The residuals of $\xi$ and the statistical model Eq. (17) versus the curvaturelength $\lambda_{2}$. (c) The statistical model Eq. (18) that predicts $\xi$ depending on the Porod length $\lambda_{1}$ and the curvature-length $\lambda_{2}$. 


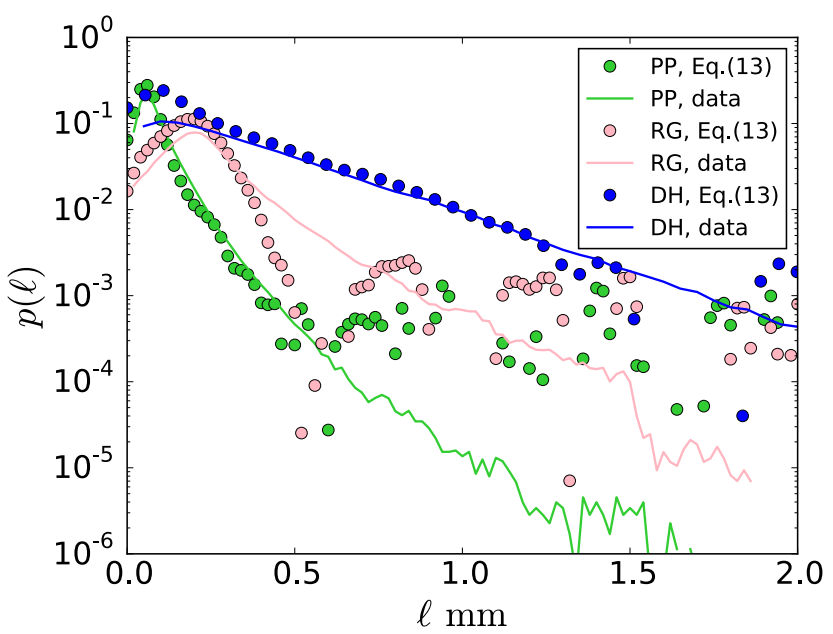

Figure 4. Comparison of the chord length distributions computed from Eq. (13) (symbols) and by direct analysis of the $\mu \mathrm{CT}$ data (solid line) for three examples of snow types (PP, RG and DH).

form (i.e., single maximum), the location of the maximum and the width of the distribution are correctly predicted by Eq. (13). However, there are obvious shortcomings, such as the oscillatory tail for the RG example when the chord length distribution is derived via Eq. (15). We will revisit these characteristics in the discussion.

\subsection{Relating the second moment of the chord length distribution to the Porod length and the curvature length}

Using the previous results we can derive an approximate relation between the second moment of the chord length distribution and the interfacial curvatures. To motivate a statistical model, we start from Eq. (14):

$\frac{\mu_{2}}{2 \mu_{1}}=f\left(\phi, \lambda_{1}, \lambda_{2}, \ldots\right)$.

We investigate the dependency of the function $f$ on parameters $\lambda_{1}, \lambda_{2}$ and $\phi$ of this expression by successively including $\lambda_{1}, \lambda_{2}$ and $\phi$ in a statistical model. In a first step we approximate $f$ by a statistical model including only $\lambda_{1}$ :

$\frac{\mu_{2}}{2 \mu_{1}}=l_{0}+l_{1} \lambda_{1}$.

The optimal parameters for model Eq. (20) are $l_{0}=-2.40 \times$ $10^{-2} \mathrm{~mm}$ and $l_{1}=1.25$, with negligible $p$ values and a correlation coefficient of $R^{2}=0.898$. The results are shown in Fig. 5a.

In view of the inclusion of the curvature-length $\lambda_{2}$, we analyzed the residuals of the previous statistical model and plotted them as a function of $\lambda_{2}$ (Fig. 5b). The correlation coefficient $\left(R^{2}=0.295\right)$ is small but including $\lambda_{2}$ in the analysis further improves the fit. The respective statistical model

$\frac{\mu_{2}}{2 \mu_{1}}=n_{0}+n_{1} \lambda_{1}+n_{2} \lambda_{2}$ (a)

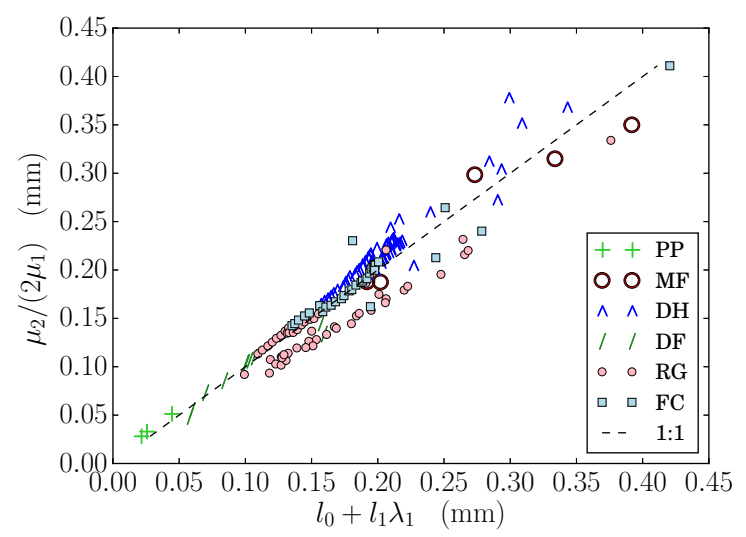

(b)

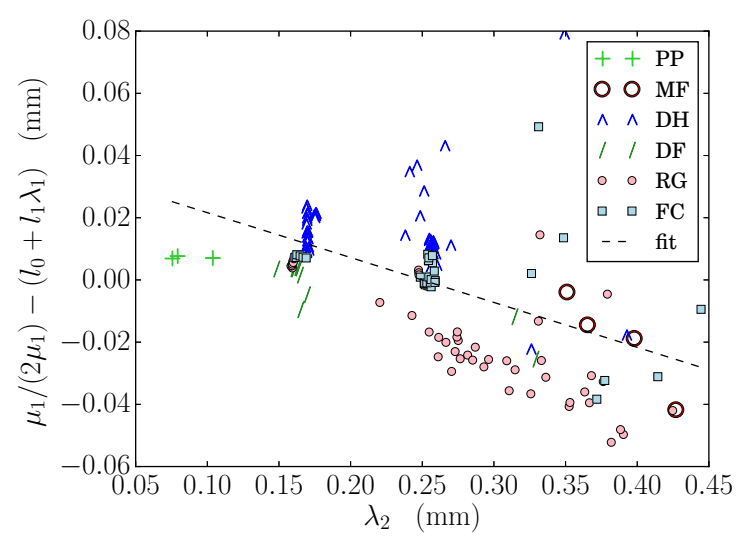

(c)

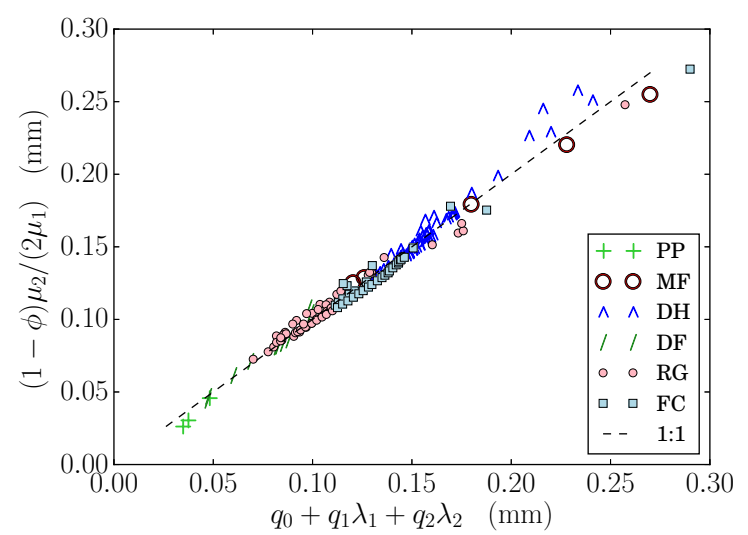

Figure 5. Scatterplots of (a) the statistical model see Eq. (20) predicting $\mu_{2} / 2 \mu_{1}$ depending on the Porod length $\lambda_{1}$; (b) the residuals of $\mu_{2} / 2 \mu_{1}$ and the statistical model Eq. (20) versus the curvature-length scale parameter $\lambda_{2}$; (c) the statistical model predicting $(1-\phi) \mu_{2} / 2 \mu_{1}$ (see Eq. 22) depending on the Porod length $\lambda_{1}$ and the curvature-length $\lambda_{2}$. 
yields optimal parameters $n_{0}=-3.95 \times 10^{-3} \mathrm{~mm}, n_{1}=1.50$ and $n_{2}=-2.46 \times 10^{-1}$ with a correlation coefficient $R^{2}=$ 0.949 . The $p$ value for the intercept $n_{0}$ is 0.36 . For $n_{1}$ and $n_{2}$ the $p$ values are again very low.

We have heuristically found a possibility of improving Eq. (21) even further. This was achieved by including a factor $(1-\phi)$ on the left-hand side. More precisely, we tried

$$
\frac{(1-\phi) \mu_{2}}{2 \mu_{1}}=q_{0}+q_{1} \lambda_{1}+q_{2} \lambda_{2}
$$

as a statistical model. Here the optimal parameters are $q_{0}=$ $-1.23 \times 10^{-2} \mathrm{~mm}, q_{1}=1.03$ and $q_{2}=-1.98 \times 10^{-1}$. The $p$ values for all coefficients are negligible and the correlation coefficient is $R^{2}=0.980$. The results are shown in Fig. 5c.

\subsection{Relating microwave metrics and optical metrics}

In the previous sections we found a statistical relation between the exponential correlation length $\xi$ and the geometrical lengths $\lambda_{1}$ and $\lambda_{2}$ on one hand and a relation between the first and second moment of the chord length distribution $\left(\mu_{1}\right.$ and $\left.\mu_{2}\right)$ and $\lambda_{1}$ and $\lambda_{2}$ on the other hand. Both findings can be recast into a direct connection between the moments of the chord lengths $\mu_{1}$ and $\mu_{2}$ and the exponential correlation length $\xi$. We express this relation in the statistical model

$\xi=c_{0}+c_{1}(1-\phi) \mu_{1}+c_{2} \frac{(1-\phi) \mu_{2}}{2 \mu_{1}}$.

Note that $(1-\phi) \mu_{1}=\lambda_{1}$ by virtue of Eq. (12), which means that we essentially replace $\lambda_{2}$ by $(1-\phi) \mu_{2} / 2 \mu_{1}$ in the statistical model Eq. (18) that relates $\xi$ to $\lambda_{1}$ and $\lambda_{2}$. We obtained the correlation coefficient $R^{2}=0.985$ for the optimal parameters $c_{0}=9.28 \times 10^{-3} \mathrm{~mm}, c_{1}=-7.53 \times 10^{-1}$ and $c_{2}=2.00$. This final relation, Eq. (23), significantly improves both models Eq. (17) and Eq. (18).

The summary of all models is given in Table 1 . To ensure that the inclusion of an additional parameter, for example by going from model Eq. (17) to model Eq. (18), is indeed an improvement, we have employed the Akaike information criterion (AIC) (Akaike, 1998). The AIC measure allows us to discern whether the improvement of the correlation coefficient is trivially caused by an increasing number of fit parameters or an actual improvement on the likelihood of the fit due to the relevance of the added parameters. Absolute AIC measures have no direct meaning, but a decrease of at least $2 k$ between two models, where $k$ is the number of extra parameters, implies a statistical improvement. For our case $k=1$ the difference in the AIC measure between Eq. (17) and Eq. (18) is 177, confirming the statistical relevance significance of $\lambda_{2}$.

\subsection{Shape factors $g^{\mathrm{G}}$ and $B$}

As an application of the values obtained for the moments of the chord length distribution we can now compute the

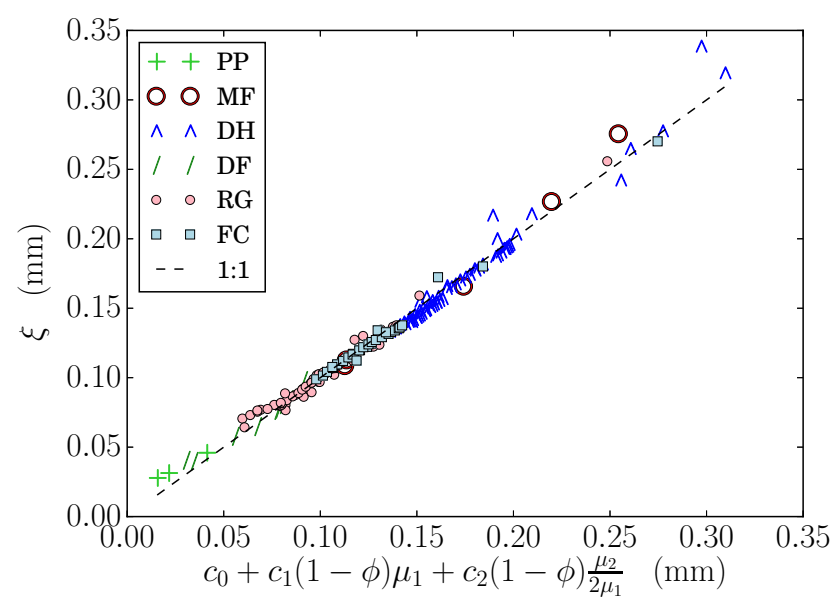

Figure 6. Scatterplot of the exponential correlation length $\xi$ versus the statistical model Eq. (23) that depends on the first and second moment of the chord length distribution, $\mu_{1}$ and $\mu_{2}$.

"shape diagram" of the optical parameters $\left(g^{\mathrm{G}}, B\right)$ suggested in Libois et al. (2013) (derived from Malinka, 2014, Eq. 60 and Eq. (A4). The results depend on the value of the Laplace transform at the absorption coefficient $\alpha$ and thus on wavelengths. For most wavelengths in the visible and nearinfrared regime $\alpha \mu_{1} \ll 1$ is small and therefore the Laplace transform Eq. (9) can be approximated by a few terms in the expansion Eq. (10). Taking typical values for $\alpha$ allows us to estimate the relative importance $\alpha \mu_{2} / 2 \mu_{1}$ of the secondorder term compared to the first-order term in the expansion Eq. (10). These values are obtained by using the values for $\kappa$ provided by Warren and Brandt (2008). The first-order $\alpha \mu_{1}$ and ratio $\alpha \mu_{2} / 2 \mu_{1}$ are calculated for typical wavelengths and shown in Table 2 . The values and standard deviations denote averages taken over all samples. Wavelengths are selected to match common optical methods, namely $0.9 \mu \mathrm{m}$ (Matzl and Schneebeli, 2006), 1.31 $\mu \mathrm{m}$ (Arnaud et al., 2011) and the shortwave infrared wavelengths $1.63,1.74$ and $2.26 \mu \mathrm{m}$ used by Domine et al. (2006). We added the wavelength $2.00 \mu \mathrm{m}$, which is not used by any instrument but has the highest value for $\alpha$ in this range. Note that for this wavelength $\alpha \mu_{1}$ is not small and the expansion of the Laplace transform, Eq. (10), likely not a good approximation. The standard deviations are high as a result of the variations due to grain type. The lowest values of $\alpha \mu_{2} / 2 \mu_{1}$ are found for fresh snow (PP) and highest for $\mathrm{DH}$ and MF.

The values in Fig. 7 for $g^{\mathrm{G}}$ and $B$ are computed for wavelength $1.3 \mu \mathrm{m}$ and shown as a scatterplot of $B$ versus $1-g^{\mathrm{G}}$ similar to Libois et al. (2013). The range of values for $B \in[1.54,1.72]$ and $\left(1-g^{\mathrm{G}}\right) \in[0.315,0.335]$ is within the range $B \in[1.25,2.09]$ and $\left(1-g^{\mathrm{G}}\right) \in[0.2,0.5]$ obtained by ray-tracing simulations for different geometrical shapes (Libois et al., 2013). The variations of the values for different snow types is, however, very small. To complete the analysis we have computed $g^{\mathrm{G}}$ and $B$ for higher absorbing 
Table 1. Summary of statistical models.

\begin{tabular}{llll}
\hline Model & Eq. & Parameters (in order) & (adj.) $R^{2}$ \\
\hline$\xi=a_{0}+a_{1} \lambda_{1}$ & $(17)$ & $5.93 \times 10^{-2} \mathrm{~mm}, 0.79$ & 0.731 \\
$\xi=b_{0}+b_{1} \lambda_{1}+b_{2} \lambda_{2}$ & $(18)$ & $1.23 \times 10^{-2} \mathrm{~mm}, 1.32,-3.85 \times 10^{-1}$ & 0.922 \\
$\xi=c_{0}+c_{1}(1-\phi) \mu_{1}+c_{2}(1-\phi) \mu_{2} / 2 \mu_{1}$ & $(23)$ & $9.28 \times 10^{-3} \mathrm{~mm},-7.53 \times 10^{-1}, 2.00$ & 0.985 \\
$\mu_{2} / 2 \mu_{1}=l_{0}+l_{1} \lambda_{1}$ & $(20)$ & $-2.40 \times 10^{-2} \mathrm{~mm}, 1.25$ & 0.898 \\
$\mu_{2} / 2 \mu_{1}=n_{0}+n_{1} \lambda_{1}+n_{2} \lambda_{2}$ & $(21)$ & $-3.95 \times 10^{-3} \mathrm{~mm}, 1.50,-2.46 \times 10^{-1}$ & 0.949 \\
$(1-\phi) \mu_{2} / 2 \mu_{1}=q_{0}+q_{1} \lambda_{1}+q_{2} \lambda_{2}$ & $(22)$ & $+1.23 \times 10^{-2} \mathrm{~mm}, 1.03,-1.98 \times 10^{-1}$ & 0.980 \\
\hline
\end{tabular}

Table 2. Determination of the absorption coefficient $\alpha$ (Warren and Brandt, 2008), the first order, the fraction of the first and second order of Eq. (10) and the obtained estimates for $B$ and $g^{\mathrm{G}}$ averaged over all snow samples, including the standard deviation $\sigma$.

\begin{tabular}{lrrrrc}
\hline Wavelength $(\mu \mathrm{m})$ & $\alpha\left(\mathrm{m}^{-1}\right)$ & $\alpha \mu_{1} \pm \sigma$ & $\mu_{2} / 2 \mu_{1} \alpha \pm \sigma(\%)$ & $B$ & $1-g^{\mathrm{G}}$ \\
\hline 0.90 & 4.1 & $0.00094 \pm 0.0003$ & $<0.5$ & $1.71 \pm 0.00$ & $0.323 \pm 0.000$ \\
1.31 & $1.2 \times 10^{2}$ & $0.026 \pm 0.008$ & $2 \pm 1$ & $1.64 \pm 0.02$ & $0.316 \pm 0.000$ \\
1.63 & $2.0 \times 10^{3}$ & $0.45 \pm 0.14$ & $37 \pm 13$ & $0.89 \pm 0.20$ & $0.253 \pm 0.011$ \\
1.74 & $1.1 \times 10^{3}$ & $0.24 \pm 0.079$ & $20 \pm 7$ & $1.19 \pm 0.14$ & $0.272 \pm 0.010$ \\
$2.00^{*}$ & $9.4 \times 10^{3}$ & $2.1 \pm 0.68$ & $172 \pm 60$ & - & - \\
2.26 & $1.1 \times 10^{3}$ & $0.25 \pm 0.08$ & $20 \pm 7$ & $1.14 \pm 0.13$ & $0.240 \pm 0.010$ \\
\hline
\end{tabular}

* Wavelength is not used for optical measurements

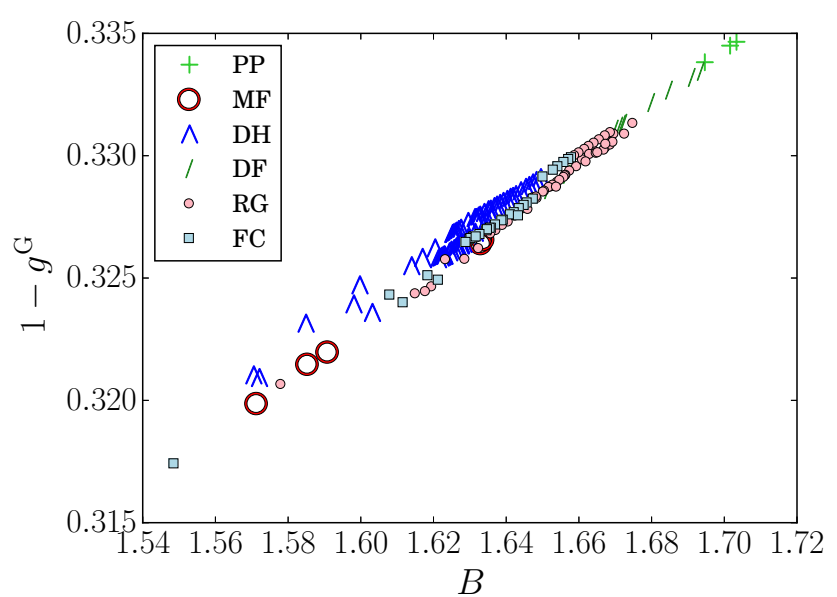

Figure 7. Scatterplot of (one minus) the asymmetry factor $g^{\mathrm{G}}$ and the optical shape factor $B$ evaluated for the refractive index at wavelength $\lambda=1.3 \mu \mathrm{m}$.

wavelengths for which the shape signature might be higher but the expansion of Eq. (10) is less reliable. The results are averaged over all snow samples and included in Table 2.

\section{Discussion}

\subsection{Methodology}

Before turning to the discussion of physical implications of the results, we first address methodological details. Retriev- ing parameters from $\mu \mathrm{CT}$ images must be taken with care. In addition to the uncertainties related to filtering and segmentation pointed out by Hagenmuller et al. (2016), the present method also requires us to discuss the interface smoothing for the validation of $\lambda_{1}$ and $\lambda_{2}$, the image resolution and the anisotropy of the samples.

\subsubsection{Geometrical interpretation}

The present analysis and cross validation of the curvature metric imposes requirements on the smoothness of the interface. The subtle influence of the smoothing parameter on the surface area $s$ and averaged mean and Gaussian curvatures $\bar{H}$ and $\bar{K}$ is apparent from Fig. 2. Naturally, $\overline{H^{2}}$ is most sensitive to smoothing. We found a competing performance of $\lambda_{1}$ and $\lambda_{2}$ with the smoothing parameter when comparing the triangulation-based estimates with the two-point correlationfunction-based values. The agreement for the surface area seems to be best with smoothing parameter $S=50$. In contrast, more smoothing is required to obtain an agreement for the curvature length. This higher sensitivity on the smoothing parameter is reasonable, since curvatures are defined by surface gradients which are more sensitive to a smooth mesh representation than the surface area. The competing behavior is caused by the smoothing filter, which preserves neither the volume nor the surface area of the enclosed ice upon smoothing iterations. This causes the drop in agreement for $\lambda_{1}$ in Fig. 2 (left, middle) with increased smoothing. As a remedy, more sophisticated smoothing filters could be used which, for example, ensure the conservation of the enclosed 
volume (Kuprat et al., 2001). Such problems could be partly avoided by computing normal vector fields and curvatures directly from voxel-based distance maps (Flin et al., 2005). A detailed comparison of all these different methods, however, is beyond the scope of this paper. In contrast to $\lambda_{1}$ and $\lambda_{2}$, the interpretation of first and second moments of the chord length distribution, $\mu_{1}$ and $\mu_{2}$, is rather straightforward, where $\mu_{1}$ is directly related to the optical diameter $d_{\mathrm{opt}}$, and $\mu_{2}$ is a measure of the variations of this size metric.

\subsubsection{Resolution}

Resolution plays an important role in obtaining estimates for $\lambda_{1}$ and $\lambda_{2}$. For a $\mu \mathrm{CT}$ measurement the resolution is commonly chosen appropriately depending on snow type. While fresh snow (PP) is typically reconstructed with $10 \mu \mathrm{m}$ voxel size, MF and larger particles have larger voxel sizes of $35 \mu \mathrm{m}$ or $54 \mu \mathrm{m}$. Since we have obtained $\lambda_{1}$ and $\lambda_{2}$ with two independent methods that agree reasonably well we conclude that the resolution is generally sufficient to estimate the involved length scales. To further confirm that there is no remaining bias with resolution we assessed the ratio $\lambda_{2}$ /voxelsize. For our data, this ratio is 9.8 on average, with a standard deviation of 2.6 and no systematic difference between small and large voxel sizes, implying that $\lambda_{2}$ can be considered as equally well resolved for all snow samples.

The image resolution plays another important role in the interpretation of the expansion of the two-point correlation function. As pointed out by Torquato (2002), a missing $r^{2}$ term is generally equivalent to a smooth interface while discontinuities, like sharp edges, would lead to a second-order term. Fresh snow and depth hoar crystals are known to have these discontinuities, at least visually. However, it remains questionable if these features can be detected objectively at the micrometer scale from image analysis. In an image, discontinuities are always smeared out, virtually contributing to the third-order term.

\subsubsection{Anisotropy}

The present data set was previously used to study the anisotropic properties of snow (Löwe et al., 2013). Therefore it is necessary to elaborate on the impact of anisotropy in the present analysis which exclusively involves isotropic two-point correlation functions. It is important to note that the our analysis does not assume isotropy; it rather includes the orientational averaging in the three Cartesian directions as a part of the method. Such a procedure is principally valid for arbitrary samples. Moreover, also the geometrical interpretation of the quantities remains valid. This was rigorously shown for $\lambda_{1}$ (Berryman, 1998) which relates the slope of the two-point correlation function at the origin for arbitrary anisotropic structures after orientational averaging to the surface area per unit volume $s$. Though we did not find a mathematical proof for the corresponding statement for $\lambda_{2}$, the agreement of $\lambda_{2}^{\text {cf }}$ (obtained from the two-point correlation function, orientationally averaged) with $\lambda_{2}^{\text {vtk }}$ (obtained from direct computation of the interfacial curvatures) strongly suggests its validity. In addition, we assessed that the residuals between $\lambda_{2}^{\text {vtk }}$ (where anisotropy does not play a role) and $\lambda_{2}^{\text {cf }}$ are not correlated with anisotropy $\left(R^{2}=0.03\right)$.

Overall, we are confident that the method can be applied to arbitrary anisotropic samples to provide orientationally averaged length scales with the correct geometric interpretation with acceptable uncertainties due to image resolution.

\subsection{Linking size metrics in snow}

Accepting the methodological uncertainties, we shall now discuss our findings of the statistical analysis and their relevance for the interpretation of snow microstructure.

\subsubsection{Including size dispersity to estimate the exponential correlation length}

By construction, the exponential correlation length $\xi$ must be understood as a proxy to characterize the entire two-point correlation function with a single length scale. This single length scale contains signatures of both properties that dominate the behavior of the two-point correlation function for small arguments $\left(\lambda_{1}\right.$ and $\left.\lambda_{2}\right)$ and other properties that dominate the tail behavior of the two-point correlation function for large arguments.

To discuss the statistical relations we will start with recovering Mätzler's model (Mätzler, 2002). This statistical model covers a relation between the exponential correlation length and the optical grain size, or in their nomenclature, the correlation length. Mätzler's model predicts the slope to be $a_{1}=0.75$, which is an average of $a_{1}=0.8$ for depth hoar and $a_{1}=0.6$ for other snow types. This is consistent with our finding $a_{1}=0.79$ since we have many depth hoar samples in the data set, suggesting that grain shape has a direct influence on the statistical relation. This influence was made quantitative by including the curvature length to the statistical analysis, resulting in the statistical model Eq. (18) (Fig. 3c). The quantitative improvement on the statistical model Eq. (16) by using Eq. (18) is given by the increase in the correlation coefficient from $R^{2}=0.733$ to $R^{2}=0.922$.

In addition we established a new statistical relation Eq. (23) between $\xi$ and the moments of the chord length distribution, $\mu_{1}$ and $\mu_{2}$. This model performs even better when the correlation coefficient $R^{2}=0.985$ is taken as a quality measure. We confirmed that the inclusion of an additional parameter in Eq. (18) and Eq. (23) indeed improves on Eq. (16), by employing the AIC measure (Akaike, 1998).

All proposed statistical models show an improvement to Eq. (1), indicating that at least two different length scales $\lambda_{1}$ and $\lambda_{2}$ or $\mu_{1}$ and $\mu_{2}$ are required to obtain a reasonable prediction of the exponential correlation length. While $\lambda_{1}$ and $\mu_{1}$ are both trivially related to the optical radius via Eq. (7) 
and Eq. (12), the two other size metrics $\mu_{2}$ or $\lambda_{2}$ are the origin of performance increase.

This seems surprising at first sight. Why should local aspects of the interface $\left(\lambda_{1}\right.$ and $\left.\lambda_{2}\right)$ determine the non-local decay of structural correlations $(\xi)$ ? To illustrate our explanation for this finding, we resort to a particle picture and consider a dense, random packing of monodisperse hard spheres. For such a packing, the particle "shape" is trivial and fully determined by the sphere diameter $d$, which determines the slope of the two-point correlation function at the origin. However, also particle positions and thus the decay of correlations is fixed by $d$. This becomes obvious from the representation $C(r)=n v_{\text {int }}(r)+n^{2} v_{\text {int }}(r) * h(r)$ for the two-point correlation function for such a system at number density $n$ (Löwe and Picard, 2015). In this representation, the spherical intersection volume $v_{\text {int }}$ and the statistics of particle positions $h(r)$ both depend on $d$. Now imagine that each sphere is deformed by a hypothetical, volume-conserving reshape operation to an irregular, non-convex particle, which is still located at the center of the original sphere. Due to reshaping, the parameter $\overline{H^{2}}$ would increase. After the reshape, neighboring particles would overlap (on average), since their maximum extension must have been increased compared to the sphere diameter. To recover a non-overlapping configuration, all particle positions must be dilated. The latter, however, also affects the tail of the two-point correlation function. This is exactly what we observe: the "shape of structural units" in snow, as exemplified by $\overline{H^{2}}$, is always correlated with the "position of the structural units" in space. We note that this particle analogy has clear limitations and only serves here to illustrate the rather abstract statistical relations between different length scales. Snow remains a bi-continuous material where individual particles cannot be distinguished by $\mu \mathrm{CT}$.

Overall, we conclude that both, $\lambda_{2}$ or $\mu_{2}$ can be used to significantly improve estimates of $\xi$ when compared to optical diameter-based estimates.

\subsubsection{Linking moments of the chord length distributions to Porod and curvature length}

Hitherto no geometrical interpretation for the second moment $\mu_{2}$ of the chord length distribution was known. Our results suggest an empirical relation, Eq. (22), that involves the two geometrical length scales $\lambda_{1}$ and $\lambda_{2}$. In the following we provide supporting arguments for the link between $\mu_{2}$ and $\lambda_{1}$ and $\lambda_{2}$ by discussing the relation Eq. (13) between the chord length distribution and the two-point correlation function.

The relation Eq. (13) was originally raised in the context of small angle scattering a long time ago (Méring and Tchoubar, 1968) and later revisited, for example by Levitz and Tchoubar (1992), revealing two different approximation steps. A first simplification comes from the assumption that consecutive chords on the random ray in Fig. 1 are statistically independent. This issue has been discussed in detail also by Roberts and Torquato (1999), who established an exact relation between the Laplace transforms of the twopoint correlation function, the chord length distribution and a surface-void correlation function based on this assumption. Their results, however, show that for level-cut Gaussian random fields, where this assumption is violated, the prediction of the chord length distribution can be still very accurate. This indicates that assuming independent chords is per se not a serious limitation. Secondly, Eq. (13) is actually an approximation for dilute systems which is generally not valid for snow.

To test the range of validity of the relation (13) for snow, we have taken three samples and computed the chord length distribution directly to compare them to the prediction of Eq. (13) as shown in Fig. 4. An obvious drawback of Eq. (13) can be seen for the RG sample. Due to the quasi-oscillations in the two-point correlation function (cf. Löwe et al., 2011), $A(\ell)$ and its second derivative assume negative values, which would imply negative values for $p(\ell)$ via Eq. (13). This is in contradiction to the meaning of $p(\ell)$ as a probability density and likely a consequence of the assumptions which are not valid for snow. Despite this obvious drawback, Fig. 4 shows that Eq. (13) yields three qualitatively consistent results for different snow types where the basic features of the chord length distribution are well predicted: first, it captures the considerable variations of the position of the maximum, the width and decay of the chord length distribution. Secondly, the relation Eq. (13) predicts that the chord length distribution tends to zero for small values, i.e., $p(0)=0$ (as confirmed in Fig. 4). This is a direct consequence of a smooth interface as shown in Wu and Schmidt (1971). Thirdly, it leads to Eq. (14), which involves the integral over the twopoint correlation function. The latter indicated a connection between $\mu_{2}, \lambda_{1}$ and $\lambda_{2}$, which was confirmed quantitatively via Eq. (21). Given the assumptions discussed above, it is not surprising that a heuristic improvement could be achieved by including a term $(1-\phi)$ in Eq. (22), since snow is not a dilute particle system and corrections containing $\phi$ terms are to be expected.

Overall, our analysis confirms that both approaches to microstructure characterization, via two-point correlation functions (with metrics $\lambda_{1}, \lambda_{2}$ ) or via chord length distribution (with metrics $\mu_{1}, \mu_{2}$ ), are not independent. They describe slightly different but interrelated structural properties which are now discussed in view of grain shape.

\subsection{Grain shape}

\subsubsection{Grain shape, a geometrical interpretation}

The international classification for seasonal snow on the ground (Fierz et al., 2009) considers grain shape as the morphological classification into snow types. This is motivated by the common but loose perception of shape as the basic geometrical form of constituent particles. It is clear that grain shape remains a vague concept unless it is formulated 
in terms of quantities which are unambiguously defined on the 3-D microstructure.

Local curvatures are often regarded as shape parameters and used to characterize snow on a more fundamental level. The relevance of the mean curvature is described and analyzed in detail in Calonne et al. (2015), where morphological transitions (e.g, faceting) of snow during temperature gradient metamorphism are visible in the distribution of mean curvatures. The present description of grain shape in snowpack models (Lehning et al., 2002; Vionnet et al., 2012) is based on a parameter called sphericity which varies between 0 and 1 for different snowtypes. This shape parameter was later given a more precise definition by Lesaffre et al. (1998) in terms of the variance of the positive mean curvatures. There were attempts to measure the sphericity from digital photographs as described by Lesaffre et al. (1998) and Bartlett et al. (2008). This definition is valid only in two dimensions and therefore difficult to compare directly to their 3-D counterparts in Calonne et al. (2015).

It is therefore natural to use objective measures such as the mean and Gaussian curvature $\bar{H}$ and $\bar{K}$ to quantify shape. Though $\bar{K}$ is computed from local properties of the interface, it has a strict topological meaning due to its relation to the Euler characteristic, which is by definition strictly independent of local shape variations of the ice-air interface. The Euler characteristic was, for example, used by Schleef et al. (2014) to characterize microstructural changes during densification. We found, however, that the contribution $\bar{K} / 3$ in $\lambda_{2}$ from Eq. (8) ranges from 1 to $13 \%$ and is on average $3.7 \%$ of $\overline{H^{2}}$. Hence the curvature-length $\lambda_{2}$ is dominated by the second moment $\overline{H^{2}}$ and thus closely related to the variance of an (inverse) size distribution, the distribution of mean curvatures. This indicates the formal similarity to $\mu_{2}$, which is also a second moment of a size distribution, the chord length distribution. Hence, both metrics can be regarded as accounting for size dispersity in snow.

Overall, we suggest that both parameters, $\mu_{2}$ and $\lambda_{2}$, can be used to objectively define a grain shape for 3-D microstructures which is closely connected to size dispersity and which naturally extends grain size (optical diameter), determining $\mu_{1}$ or $\lambda_{1}$. Note that within this definition, grain shape is not a dimensionless parameter. With this perception of shape we now connect back to the original applications of microwave and optical modeling.

\subsubsection{Grain shape for microwave modeling}

Thus far, the exponential correlation length $\xi$ as a key parameter for MEMLS-based microwave modeling was mainly predicted from the optical diameter. Our conclusions from Sect. 5.2.1 could now be restated: the inclusion of a grain shape parameter, $\lambda_{2}$ or $\mu_{2}$ improves the prediction of the exponential correlation length significantly. Otherwise, according to the conclusion from the previous section, one may alternatively restate that size dispersity has an influence on mi- crowave properties. This is known from models other than MEMLS, where an influence of polydispersity on the effective grain scaling parameter within DMRT-ML microwave modeling was found (Roy et al., 2013).

This equivalence of shape and size dispersity at the level of two-point correlation functions can be further illustrated by an interesting example. Consider a microstructure of polydisperse spherical particles. The definition of grain shape from the classification (Fierz et al., 2009) would assign a spherical shape to this microstructure, while the averaged squaredmean curvature $\overline{H^{2}}$ would instead vary depending on the variance of particle radii. As pointed out by Tomita (1986), for low density such a system of polydisperse spherical particles can always be mapped uniquely onto an assembly of monodisperse but irregularly shaped particles by solving an integral equation, if only the two-point correlation function is considered. Shape can be equivalent to polydispersity, and snow types which are visually very different might still have very similar physical properties. This example also explains why the objective size dispersity parameters $\lambda_{2}$ or $\mu_{2}$ cannot be mapped onto the classical definition of grain type from Fierz et al. (2009).

\subsubsection{Grain shape in geometrical optics}

Finally, we turn to the implications of size dispersity or grain shape on geometrical optics within the scope of Malinka (2014) based on chord length distributions.

As pointed out by Malinka (2014), if consecutive chords were statistically independent, i.e., a Markovian process, then the obtained distribution would be an exponential, and all optical properties solely determined by the optical diameter (or $\mu_{1}$ ). To quantify the deviation from an exponential chord length distributions we calculated the fraction $\mu_{2} / 2 \mu_{1}^{2}$, which is unity for a exponential chord length distribution. This fraction is on average 0.75 for $\mathrm{RG}, 0.76$ for $\mathrm{MF}, 0.77$ for PP and DF, 0.79 for FC and the closest value to unity is 0.876 for $\mathrm{DH}$. This implies that the chord length distribution for depth hoar is closest to an exponential, which can be visually confirmed by Fig. 4 . We reach a similar conclusion for the two-point correlation function where $\lambda_{1}$ is already a fairly good predictor for the exponential correlation length when depth hoar is considered (see Fig. 3a). However, due to the deviations from an exponential distribution, an influence of shape via $\mu_{2}$ on the optical properties would be expected according to Malinka (2014).

Using the chord length distributions we were able to calculate the shape factors $B$ and $g^{\mathrm{G}}$ from Malinka (2014) and Libois et al. (2013) in the limit of low absorption where both approaches can be compared. The $\left(B, g^{\mathrm{G}}\right)$ shape diagram (cf. Fig 1a in Libois et al., 2013) in Fig. 7 was obtained for wavelength $1.3 \mu \mathrm{m}$ where the Laplace transform Eq. (10) can be approximated by the first and second order. The variations of the absolute values for $B, g^{\mathrm{G}}$ shown in Fig. 7 predominantly stem from corrections which are linear in $\mu_{1}$ (by virtue of 
Eq. A5), while the small, scattered deviations from a perfect straight line are caused by $\mu_{2}$. If $B$ and $g^{\mathrm{G}}$ were evaluated for wavelength $0.9 \mu \mathrm{m}$, the influence of $\mu_{2}$ would be even smaller. Our results show that the values for $B$ and $g^{\mathrm{G}}$ are exactly within the range that is suggested by ray-tracing simulations for various geometrical shapes for a wavelength of $0.9 \mu \mathrm{m}$ (Libois et al., 2013), but show a much smaller variation over the entire set of snow samples. Comparing our results to ray tracing of geometrical shapes is, however, not straightforward, since the 3-D microstructures cannot be mapped on an ensemble of regular geometrical objects.

The predicted values for $B$ (Fig. 7) are very similar to the values obtained by experiments (Libois et al., 2014) but show a smaller variation. It should be noted that, as the authors discuss, the correlation between the experimentally obtained $B$ and shape, as defined by Fierz et al. (2009), is statistically not significant and variations might be attributed to shadowing effects relevant at higher densities.

Overall, our analysis indicates a smaller variation of optical properties with shape via $\mu_{2}$ according to Malinka (2014) when compared to other methods. We can only hypothesize potential origins which are connected to the present analysis. A crucial assumption made in the geometrical optics framework (Malinka, 2014) is the statistical independence of the chord length and the consecutive ice-air incidence angle for a ray which passes through a grain. Such an assumption might be progressively violated for lower absorption where a higher number of internal reflections in fact probes this assumption more often. Hence the true effect of shape on $B$ and $g^{\mathrm{G}}$ might be more pronounced than predicted by size dispersity via $\mu_{2}$ (Malinka, 2014). Further details on the discrepancies between measurements, simulations and theory remain to be elucidated by combining tomography imaging and shape analysis together with optical measurements and ray-tracing simulations in the future.

\section{Conclusions}

We have analyzed different microstructural length scales $\left(\lambda_{1}\right.$, $\lambda_{2}$ and $\mu_{1}, \mu_{2}$ ) of snow samples which were derived from the two-point correlation function and chord length distribution, respectively. All length scales have a well-defined geometrical meaning. While the first-order quantities $\left(\mu_{1}, \lambda_{1}\right)$ are both related to the mean size (optical equivalent diameter), their higher-order counterparts $\left(\lambda_{2}, \mu_{2}\right)$ are objective measures of size dispersity present in the snow microstructure.
For the two-point correlation function, the length scale $\lambda_{2}$ is essentially determined by the second moment of the mean curvature distribution. For the chord lengths, $\mu_{2}$ is the second moment of the chord length distribution. Both quantities naturally extend the concept of mean grain size as covered by the optical equivalent diameter. The statistical relation established between $\lambda_{1}, \lambda_{2}$ and $\mu_{1}, \mu_{2}$ indicates that in practice the two measures of size dispersity can be used interchangeably.

We have argued that size dispersity is one possible route towards an objective definition of grain shape, and thus both quantities $\left(\lambda_{2}, \mu_{2}\right)$ can be regarded as measures of shape. Within this interpretation, we found that grain shape or size dispersity significantly improves a widely used statistical model for the exponential correlation length (as a key size metric for MEMLS-based microwave modeling).

We have also used this interpretation of shape to assess the so-called optical shape factor $B$ which can be related to $\mu_{1}$ and $\mu_{2}$ in the framework of Malinka (2014). The results suggest that size dispersity is only a first, but likely not a complete step, to characterize shape for optical modeling.

Overall, defining grain shape via dispersity measures $\mu_{2}$ or $\lambda_{2}$ provides a clear intersection between microwave modeling of snow (if based on the exponential correlation length) and optical modeling of snow (if based on Malinka, 2014). We do not believe this intersection to be exhaustive: the influence of shape in snow optics likely involves more than size dispersity. Additionally, size dispersity is likely not sufficient to explain the full diversity of microwave properties of snow. However, the established overlap of relevant microstructure parameters provides a clear quantitative starting point for further improvements.

\section{Data availability}

The length-scale data required to reproduce all statistical models with corresponding figures $(2,3,5,6,7)$ can be found in the Supplement. The underlying 3-D image data can be obtained through the corresponding author. 


\section{Appendix A: Optical shape factor $B$ from moments of the chord length distribution}

To derive an expression of the optical shape factor $B$ in terms of the moments of the chord length distribution, we start from expression (Libois et al., 2013, Eq. 6) for the single scattering co-albedo

$$
(1-\omega)=B \frac{\gamma V}{2 \Sigma},
$$

which is related to $B$, the average volume of a particle $V$, the average projected area of a particle $\Sigma$ and the absorption coefficient $\gamma$. This can be recast in terms of the mean chord length using (Malinka, 2014, Eq. 6), which yields, adopting the notation of the present paper, the relation

$$
(1-\omega)=B \frac{\alpha \mu_{1}}{2}
$$

However, an expression for the single scattering co-albedo is directly provided by Malinka (2014, Eq. 56). Inserting (Malinka, 2014, Eqs. 29, 42, 49, 18) and rearranging terms we obtain

$$
(1-\omega)=\frac{T_{\text {out }}(n)}{1+\frac{T_{\text {out }}(n)}{n^{2}} \frac{\widehat{p}(\alpha)}{1-\widehat{p}(\alpha)}}
$$

in terms of the real part of the refractive index $n$, the averaged Fresnel transmittance coefficient $T_{\text {out }}(n)$ (given by Malinka (2014, Eq. 19) in closed form) and the Laplace transform of the chord length distribution $\widehat{p}(\alpha)$.
To obtain an expression for $B$ by comparing Eq. (A2) and Eq. (A3) it must be noted that both expression are based on slightly different assumptions. While Eq. (A1) is meant to be valid only in the limit of low absorption (Libois et al., 2013), Eq. (A3) is valid for arbitrary values of $\alpha$. This is reflected by the existence of the limit $\alpha \rightarrow \infty$ in Eq. (A3), while Eq. (A2) diverges if $B$ is regarded as a constant which is strictly independent of $\alpha$. Hence the comparison of Eq. (A2) and Eq. (A3) must be limited to small values of $\alpha \mu_{1}$ in order to obtain an expression for $B$ which can be compared to the results from (Libois et al., 2013). That said, we equate Eq. (A2) and Eq. (A3), take into account an additional factor of 2 between Malinka (2014) and Libois et al. (2013) due to a different treatment of the extinction efficiency, and we end up with

$$
B=\frac{1}{\alpha \mu_{1}} \frac{T_{\text {out }}(n)}{1+\frac{T_{\text {out }}(n)}{n^{2}} \frac{\widehat{p}(\alpha)}{1-\widehat{p}(\alpha)}} .
$$

Complemented by the approximation Eq. (10) for the Laplace transform $\widehat{p}$, the expression (Malinka, 2014, Eq. 19) for $T_{\text {out }}(n)$, this yields an expression of the shape factor $B$ in terms of the first and second moments, $\mu_{1}$ and $\mu_{2}$, of the chord length distribution, the real part of the refractive index $n$ and the absorption coefficient $\alpha$.

To explicitly reveal the correction of $B$ for small $\alpha$ which involves the second moment of the chord length distribution, we expand Eq. (A4) around $\alpha=0$ to obtain

$$
B=n^{2}\left[1-\left(\alpha \mu_{1}\right)\left(\frac{n^{2}}{T_{\text {out }}(n)}-1+\frac{\mu_{2}}{2 \mu_{1}^{2}}\right)\right]
$$


Acknowledgements. The authors thank G. Picard for a constructive feedback on an earlier version of the manuscript and $\mathrm{S}$. Torquato for helpful clarifications on the factor $2 / 3$ between $s_{\mathrm{mf}}$ and $s_{\text {cf }}$. M. Lehning provided valuable suggestions on the statistical methods. The comments from the reviewers Q. Libois and A. Malinka greatly helped to improve the manuscript and to shape the conclusions of the paper. The work was funded by the Swiss National Science Foundation via grant no. 200021_143839.

Edited by: Guillaume Chambon

Reviewed by: A. Malinka and Q. Libois

\section{References}

Akaike, H.: Selected papers of Hirotugu Akaike, 199-213, Springer New York, doi:10.1007/978-1-4612-1694-0_15, 1998.

Arnaud, L., Picard, G., Champollion, N., Domine, F., Gallet, J., Lefebvre, E., Fily, M., and Barnola, J.: Measurement of vertical profiles of snow specific surface area with a $1 \mathrm{~cm}$ resolution using infrared reflectance: instrument description and validation, J. Glaciol., 57, 17-29, doi:10.3189/002214311795306664, 2011.

Bartlett, S. J., Rüedi, J.-D., Craig, A., and Fierz, C.: Assessment of techniques for analyzing snow crystals in two dimensions, Ann. Glaciol., 48, 103-112, doi:10.3189/172756408784700752, 2008.

Berryman, J. G.: Planar spatial correlations, anisotropy, and specific surface area of stationary random porous media, J. Appl. Phys., 83, 1685-1693, doi:10.1063/1.366885, 1998.

Brun, E., David, P., Sudul, M., and Brunot, G.: A numerical model to simulate snow-cover stratigraphy for operational avalanche forecasting, J. Glaciol., 38, 13-22, http://www.ingentaconnect. com/content/igsoc/jog/1992/00000038/00000128/art00003, 1992.

Brzoska, J.-B., Flin, F., and Barckicke, J.: Explicit iterative computation of diffusive vapour field in the 3D snow matrix: preliminary results for low flux metamorphism, Ann. Glaciol., 48, 13-18, doi:10.3189/172756408784700798, 2008.

Calonne, N., Flin, F., Geindreau, C., Lesaffre, B., and Rolland du Roscoat, S.: Study of a temperature gradient metamorphism of snow from 3-D images: time evolution of microstructures, physical properties and their associated anisotropy, The Cryosphere, 8, 2255-2274, doi:10.5194/tc-8-2255-2014, 2014a.

Calonne, N., Geindreau, C., and Flin, F.: Macroscopic modeling for heat and water vapor transfer in dry snow by homogenization, J. Phys. Chem. B, 118, 13393-13403, doi:10.1021/jp5052535, 2014b.

Calonne, N., Flin, F., Lesaffre, B., Dufour, A., Roulle, J., Puglièse, P., Philip, A., Lahoucine, F., Geindreau, C., Panel, J.-M., Rolland du Roscoat, S., and Charrier, P.: CellDyM: A room temperature operating cryogenic cell for the dynamic monitoring of snow metamorphism by time-lapse X-ray microtomography, Geophys. Res. Lett., 42, 3911-3918, doi:10.1002/2015GL063541, 2015.

Debye, P., Anderson, H., and Brumberger, H.: Scattering by an inhomogeneous solid .2. The correlation function and its application, J. Appl. Phys., 28, 679-683, doi:10.1063/1.1722830, 1957.

Ding, K.-H., Xu, X., and Tsang, L.: Electromagnetic scattering by bicontinuous random microstructures with discrete per- mittivities, IEEE T. Geosci. Remote Sens., 48, 3139-3151, doi:10.1109/TGRS.2010.2043953, 2010.

Domine, F., Salvatori, R., Legagneux, L., Salzano, R., Fily, M., and Casacchia, R.: Correlation between the specific surface area and the short wave infrared (SWIR) reflectance of snow, Cold Reg. Sci. Technol., 46, 60-68, doi:10.1016/j.coldregions.2006.06.002, 2006.

Durand, M., Kim, E. J., and Margulis, S. A.: Quantifying uncertainty in modeling snow microwave radiance for a mountain snowpack at the point-scale, including stratigraphic effects, IEEE $\mathrm{T}$ Geosci. Remote, 46, 1753-1767, doi:10.1109/TGRS.2008.916221, 2008.

Fierz, C., Armstrong, R. L., Durand, Y., Etchevers, P., Greene, E., McClung, D. M., Nishimura, K., Satyawali, P. K., and Sokratov, S.: The international classification for seasonal snow on the ground, IHP-VII Technical Documents in Hydrology, 83, IACS Contribution (1), UNESCO-IHP, Paris, 2009.

Flin, F., Brzoska, J.-B., Coeurjolly, D., Pieritz, R., Lesaffre, B., Coleou, C., Lamboley, P., Teytaud, F., Vignoles, G. L., and Delesse, J.-F.: Adaptive estimation of normals and surface area for discrete 3D objects: application to snow binary data from X-ray tomography, Image Processing, IEEE Transactions on, 14, 585596, doi:10.1109/TIP.2005.846021, 2005.

Frisch, H. L. and Stillinger, F. H.: Contribution to the statistical geometric basis of radiation scattering, J. Chem. Phys., 38, 22002207, doi:10.1063/1.1733950, 1963.

Gille, W.: Chord length distributions and small-angle scattering, Eur. Phys. J. B, 17, 371-383, doi:10.1007/s100510070116, 2000.

Hagenmuller, P., Matzl, M., Chambon, G., and Schneebeli, M.: Sensitivity of snow density and specific surface area measured by microtomography to different image processing algorithms, The Cryosphere, 10, 1039-1054, doi:10.5194/tc-10-1039-2016, 2016.

Kokhanovsky, A. A. and Zege, E. P.: Scattering optics of snow, Appl. Opt., 43, 1589-1602, doi:10.1364/AO.43.001589, 2004.

Krol, Q. and Löwe, H.: Analysis of local ice crystal growth in snow, J. Glaciol, 62, 378-390, doi:10.1017/jog.2016.32, 2016.

Kuprat, A., Khamayseh, A., George, D., and Larkey, L.: Volume Conserving Smoothing for Piecewise Linear Curves, Surfaces, and Triple Lines, J. Comput. Phys., 172, 99-118, doi:10.1006/jcph.2001.6816, 2001.

Lehning, M., Bartelt, P., Brown, B., Fierz, C., and Satyawali, P.: A physical snowpack model for the Swiss avalanche warning: Part II. Snow microstructure, Cold Reg. Sci. Technol., 35, 147-167, doi:10.1016/S0165-232X(02)00073-3, 2002.

Leinss, S., Löwe, H., Proksch, M., Lemmetyinen, J., Wiesmann, A., and Hajnsek, I.: Anisotropy of seasonal snow measured by polarimetric phase differences in radar time series, The Cryosphere, 10, 1771-1797, doi:10.5194/tc-10-1771-2016, 2016.

Lesaffre, B., Pougatch, E., and Martin, E.: Objective determination of snow-grain characteristics from images, Ann. Glaciol., 26, 112-118, 1998.

Levitz, P. and Tchoubar, D.: Disordered porous solids : from chord distributions to small angle scattering, J. Phys. I, 2, 771-790, doi:10.1051/jp1:1992174, 1992.

Libois, Q., Picard, G., Dumont, M., Arnaud, L., Sergent, C., Pougatch, E., Sudul, M., and Vial, D.: Experimental determi- 
nation of the absorption enhancement parameter of snow, J. Glaciol., 60, 714-724, doi:10.3189/2014J0G14J015, 2014.

Libois, Q., Picard, G., France, J. L., Arnaud, L., Dumont, M., Carmagnola, C. M., and King, M. D.: Influence of grain shape on light penetration in snow, Cryosphere, 7, 1803-1818, doi:10.5194/tc-7-1803-2013, 2013.

Löwe, H. and Picard, G.: Microwave scattering coefficient of snow in MEMLS and DMRT-ML revisited: the relevance of sticky hard spheres and tomography-based estimates of stickiness, The Cryosphere, 9, 2101-2117, doi:10.5194/tc-9-2101-2015, 2015.

Löwe, H., Spiegel, J. K., and Schneebeli, M.: Interfacial and structural relaxations of snow under isothermal conditions, J. Glaciol., 57, 499-510, doi:10.3189/002214311796905569, 2011.

Löwe, H., Riche, F., and Schneebeli, M.: A general treatment of snow microstructure exemplified by an improved relation for thermal conductivity, The Cryosphere, 7, 1473-1480, doi:10.5194/tc-7-1473-2013, 2013.

Malinka, A. V.: Light scattering in porous materials: Geometrical optics and stereological approach, J. Quant. Spectrosc. Ra., 141, 14-23, doi:10.1016/j.jqsrt.2014.02.022, 2014.

Matzl, M. and Schneebeli, M.: Measuring specific surface area of snow by near-infrared photography, J. Glaciol., 52, 558-564, doi:10.3189/172756506781828412, 2006.

Mätzler, C.: Improved Born approximation for scattering of radiation in a granular medium, J. Appl. Phys., 83, 6111-6117, 1998.

Mätzler, C.: Relation between grain-size and correlation length of snow, J. Glaciol., 48, 461-466, doi:10.3189/172756502781831287, 2002.

Mätzler, C. and Wiesmann, A.: Extension of the microwave emission model of layered snowpacks to coarse-grained snow, Rem. Sens. Environ., 70, 317-325, doi:10.1016/S00344257(99)00047-4, 1999.

Méring, J. and Tchoubar, D.: Interprétation de la diffusion centrale des rayons X par les systèmes poreux. I, J. App. Crystallogr., 1, 153-165, doi:10.1107/S0021889868005212, 1968.

Michielsen, K. and Raedt, H. D.: Integral-geometry morphological image analysis, Phys. Rep., 347, 461-538, doi:10.1016/S03701573(00)00106-X, 2001.

Newman, M. and Barkema, G.: Monte carlo methods in statistical physics, Clarendon Press, 1999.

Pan, J., Durand, M., Sandells, M., Lemmetyinen, J., Kim, E. J., Pulliainen, J., Kontu, A., and Derksen, C.: Differences Between the HUT Snow Emission Model and MEMLS and Their Effects on Brightness Temperature Simulation, IEEE T Geosci. Remote, 54, 2001-2019, doi:10.1109/TGRS.2015.2493505, 2016.

Picard, G., Arnaud, L., Domine, F., and Fily, M.: Determining snow specific surface area from near-infrared reflectance measurements: Numerical study of the influence of grain shape, Cold Reg. Sci. Technol., 56, 10-17, doi:10.1016/j.coldregions.2008.10.001, 2009.

Proksch, M., Löwe, H., and Schneebeli, M.: Density, specific surface area and correlation length of snow measured by highresolution penetrometry, J. Geophys. Res.-Earth, 120, 346-362, doi:10.1002/2014JF003266, 2015a.

Proksch, M., Mätzler, C., Wiesmann, A., Lemmetyinen, J., Schwank, M., Löwe, H., and Schneebeli, M.: MEMLS3\&a: Microwave Emission Model of Layered Snowpacks adapted to include backscattering, Geosci. Model Dev., 8, 2611-2626, doi:10.5194/gmd-8-2611-2015, 2015 b.
Roberts, A. and Torquato, S.: Chord-distribution functions of three-dimensional random media: Approximate first-passage times of Gaussian processes, Phys. Rev. E, 59, 4953-4963, doi:10.1103/PhysRevE.59.4953, 1999.

Roy, A., Picard, G., Royer, A., Montpetit, B., Dupont, F., Langlois, A., Derksen, C., and Champollion, N.: Brightness Temperature Simulations of the Canadian Seasonal Snowpack Driven by Measurements of the Snow Specific Surface Area, IEEE Trans. Geosci. Remote Sens., 51, 4692-4704, doi:10.1109/TGRS.2012.2235842, 2013.

Schleef, S., Löwe, H., and Schneebeli, M.: Influence of stress, temperature and crystal morphology on isothermal densification and specific surface area decrease of new snow, The Cryosphere, 8 , 1825-1838, doi:10.5194/tc-8-1825-2014, 2014.

Schleef, S., Loewe, H., and Schneebeli, M.: Hot-pressure sintering of low-density snow analyzed by X-ray microtomography and in situ microcompression, Act. Mater., 71, 185-194, doi:10.1016/j.actamat.2014.03.004, 2014.

Tan, S., Aksoy, M., Brogioni, M., Macelloni, G., Durand, M., Jezek, K. C., Wang, T. L., Tsang, L., Johnson, J. T., Drinkwater, M. R., and Brucker, L.: Physical models of layered polar firn brightness temperatures from 0.5 to $2 \mathrm{GHz}$, IEEE J. Sel. Top. Appl., 8, 3681-3691, doi:10.1109/JSTARS.2015.2403286, 2015.

Tomita, H.: Statistical properties of random interface system, Prog. Theo. Phys, 75, 482-495, doi:10.1143/PTP.75.482, 1986.

Torquato, S.: Random heterogeneous materials: Microstructure and macroscopic Properties, Interdisciplinary Applied Mathematics, Springer, 2002.

Underwood, E. E.: Stereology, or the quantitative evaluation of microstructures, J. Microsc., 89, 161-180, doi:10.1111/j.13652818.1969.tb00663.x, 1969.

Vallese, F. and Kong, J.: Correlation-function studies for snow and ice, J. Appl. Phys., 52, 4921-4925, doi:10.1063/1.329453, 1981.

Vionnet, V., Brun, E., Morin, S., Boone, A., Faroux, S., Le Moigne, P., Martin, E., and Willemet, J.-M.: The detailed snowpack scheme Crocus and its implementation in SURFEX v7.2, Geosci. Model Dev., 5, 773-791, doi:10.5194/gmd-5-773-2012, 2012.

Warren, S. G. and Brandt, R. E.: Optical constants of ice from the ultraviolet to the microwave: A revised compilation, J. Geophys. Res.-Atmos., 113, d14220, doi:10.1029/2007JD009744, 2008.

Wiesmann, A. and Mätzler, C.: Microwave Emission Model of Layered Snowpacks, Remote Sens. Environ., 70, 307-316, doi:10.1016/S0034-4257(99)00046-2, 1999.

Wiesmann, A., Mätzler, C., and Weise, T.: Radiometric and structural measurements of snow samples, Radio Sci., 33, 273-289, 1998.

Wu, H.-I. and Schmidt, P. W.: Intersect distributions and smallangle X-ray scattering theory, J. Appl. Crystallogr., 4, 224-231, doi:10.1107/S0021889871006745, 1971.

Zurk, L., Tsang, L., Shi, J., and Davis, R.: Electromagnetic scattering calculated from pair distribution functions retrieved from planar snow sections, IEEE T. Geosci. Remote, 35, 1419-1428, doi:10.1109/36.649796, 1997. 